\title{
An Integrated Method for 3D Reconstruction, Dimensional Analysis and Manipulation of an Object Based on Image Processing
}

\author{
Soham Pinge, Nitish Wadhavkar, Amey Singh, Amol Deshpande
}

\begin{abstract}
Analysis of dimensions of a product, its visualization as a 3D model \& volume estimation, positively impact the operations of manufacturing department in an industry. In this paper, a system has been designed to integrate the proposed features like 3D reconstruction, dimensional analysis \& support for manipulating the reconstructed model. Research was carried out to estimate the volume of objects using their virtual $3 D$ model $\&$ allow the user to edit this model which later can be compared with the original object, for manipulation. The pictures of an object from different planes are captured and using image processing, it is reconstructed in $3 D$, which is used to estimate parameters like volume, surface area, maximum dimensions across different planes. This edited $3 D$ model will be utilized for generating a binary file representing the changes. This system provides detailed information to the user along with flexibility, thus enhancing the way in which the manufacturing industry works, as it requires no manual input or interaction once the process begins.
\end{abstract}

Keywords: Industrial Solutions, Mathematics and Computing, Image Processing, Volume Estimation, 3D Reconstruction, Dimensional Analysis, Edge Detection.

\section{INTRODUCTION}

In manufacturing industry, analysis of dimensions of objects is of utmost importance. And this should be carried out as efficiently and safely as possible, considering production for sensitive domains where non-destructive processes should be used. Also, with the COVID-19 pandemic, manufacturing and testing has become a huge challenge for industries with paucity of staff, further increasing the need for automation at every level. The demand for non-contact based industrial solutions has grown exponentially.

Manuscript received on April 20, 2021.

Revised Manuscript received on April 26, 2021.

Manuscript published on April 30, 2021.

* Correspondence Author

Soham Pinge*, Department of Electronics \& Telecommunications, Sardar Patel Institute of Technology, Mumbai, India. Email: soham.pinge@spit.ac.in

Nitish Wadhavkar, Department of Electronics \& Telecommunications, Sardar Patel Institute of Technology, Mumbai, India. Email: nitish.wadhavkar@spit.ac.in

Amey Singh, Department of Electronics \& Telecommunications, Sardar Patel Institute of Technology, Mumbai, India. Email: amey.singh@spit.ac.in

Dr. Amol Deshpande, Department of Electronics \& Telecommunications, Sardar Patel Institute of Technology, Mumbai, India. Email: amol deshpande@spit.ac.in

(C) The Authors. Published by Blue Eyes Intelligence Engineering and Sciences Publication (BEIESP). This is an open access article under the CC BY-NC-ND license (http://creativecommons.org/licenses/by-nc-nd/4.0/)
In today's age, automation is of prime importance. Operations like 3D Reconstruction and volume estimation are performed regularly in industries, but manually and separately. Automation will save effort and the time invested in manual execution. This project not only introduces methods for automation of the said processes but also consolidates these using step by step into codependent procedures for integrating them with each other. Therefore, this paper proposes a methodology for the improvement of the quality of industrial automation, manufacturing and testing, that uses new techniques and integrates the processes of dimensional analysis, volume estimation, 3D reconstruction and provides support for manipulation of an object, which can then be exported in the form of a standard .stl file format to be utilized as preferred by the user.

\section{LITERATURE SURVEY}

Before diving deep into the paper, we shall look at some related works. There are two main techniques for 3D reconstruction, LIDAR and photogrammetry. LIDAR uses laser to scan objects and are more accurate and convenient. But, in design and manufacturing industries, the real time images of objects are necessary as operations like logo marking, designing, colouring, etc. are involved. In such applications, photogrammetry is a suitable technique. In [1] only orthogonal projections have been considered, therefore no $3 d$ reconstruction has been performed. In [2] we see that deep learning has been implemented, which requires presence of large databases, providing a hindrance in our case because there is no existence of a useful dataset and even if there was, its storage would have further used up a lot of memory. Also, the use of a single camera is time consuming, has a high margin of error and maybe require additional complex robotics. In [3] although machine learning is not used, the method of Scale Invariant Feature Transform (SIFT) is used. This method too requires a significant number of key-points stored in a database, giving rise to the same earlier mentioned problems. In [4] a method known as Structure from motions is used. It mainly describes parameters such as effective

position and attribute calculation of the camera and our object. The smoothing of the images is not obtained upto a great ex- tent. In [5] a volume measurement system for calculating volume of tomatoes is proposed. This provides a good and fast solution, but it comes at the cost of accuracy which reaches a maximum of $80 \%$ despite capturing five different images.

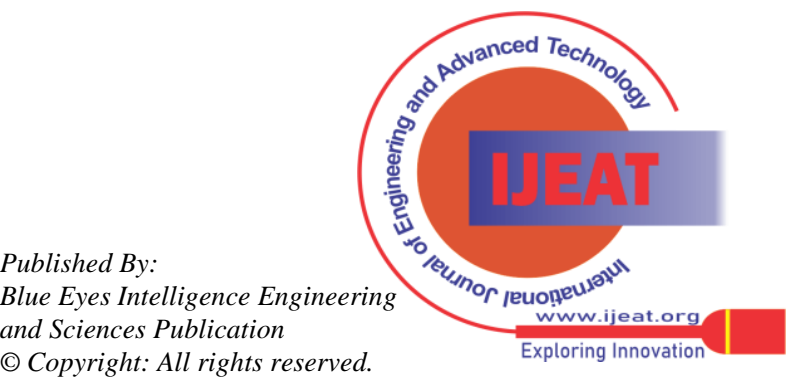


Also, this method is limited to tomatoes and cannot be applied to other objects.

In [6] a comprehensive study on various techniques and algorithms of $3 d$ reconstruction has been performed. In [7] a principle of feature detection and matching is followed prior to reconstruction. Here camera self-calibration was performed, although it has to be improved along with reconstruction of noisy data points. In [8] a concept in particle visualization has been discussed about, but it can't be scaled to larger objects. In [9] detailed analysis of all applications and challenges related to $3 \mathrm{~d}$ reconstruction. No paper talks about volume estimation, except [8] and [9]. In [10] a method for viewing $3 \mathrm{~d}$ models from $2 \mathrm{~d}$ pictures is proposed. To summarize, many of the above-mentioned papers have implemented deep learning for achieving something similar.

To implement deep learning in this project was not possible due to the paucity of complex hardware $\&$ an existing dataset consisting of a variety of shapes. Moreover, except a couple, no paper talks about volume estimation, and the ones that do mention it provide an accuracy of only about 80 to $85 \%$.

\section{METHODOLOGY}

The Methodology of this project can be divided into 5 parts:
A. Edge Detection and Filtering
B. Calibration of Camera
C. 3D Reconstruction
D. Dimensional Analysis and Volume Estimation
E. Support for Manipulation

\section{A. Edge Detection and Filtering}

This is a simple process which contains the following sub-processes:

1) Detect Edges of the object from the captured image, as shown in figure 1.

2) Filter and remove unwanted edges and noise, which can be seen in figure 2, as many images can be rendered redundant due to noise. [4] [5]

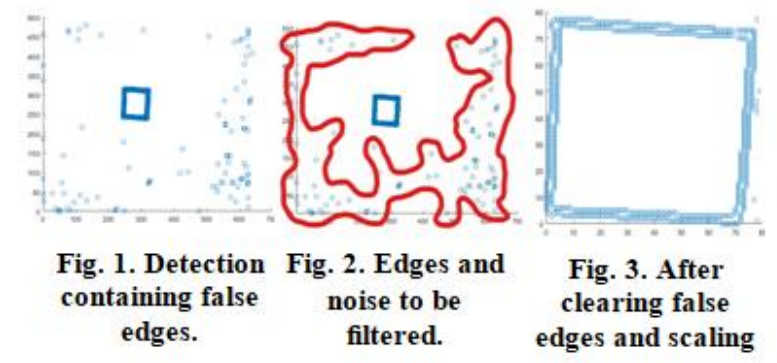

\section{B. Calibration of Camera}

Since the objects in consideration will not always be a flat surface, focal length approach for calibration fails (Since the focal length approach extracts multiple points from a flat surface to form a control matrix). For this we have used a different approach.

At a fixed distance from the camera, the ratio of any dimension of the object in the image (in pixels) to the ratio of the same dimension in reality (in cm or any convenientunit) remains constant for all objects. This is known as the pixels per $\mathrm{cm}$ ratio or PPCm (or pixels per metric in general). In this paper, we will work with the aforementioned approach. This method is detailed as follows:

1) For beginning the setting up process, we will click pictures of any object ofwhich the length is known to us. Call this the control object.

2) In reality, the value of one dimension of the control object is $\mathrm{X} \mathrm{cm}$. From thecaptured picture, the value of the same dimension is found out as $\mathrm{Y}$ pixels.

3) The pixels per $\mathrm{cm}$ ratio will be given as

PPCm $=$ Measured Dimension (in pixels)

Real Dimension (in cm)

$=\underline{Y}($ pixels $/ \mathrm{cm})$

$\mathrm{X}$

4) Now, we will find out the dimension of the unknown object. In this case we have the PPCm ratio and the measured dimension and we have to find the real dimension. Consider the measured dimension of the unknown object to be $\mathrm{Z}$ pixels, therefore the real dimension is given by:

Real Dimension $=$ Measured Dimension(in pixels) $\ldots$. (2) PPCm (in pixels/cm)

$=\underline{\mathrm{Z}}($ in $\mathrm{cm})$

$$
\frac{\mathrm{Y}}{\mathrm{X}}
$$

\section{3D Reconstruction}

1) After calibrating the camera, we will proceed with the first step in this part, i.e. capturing of images. Images will be captured from atleast 3 different angles. [2] [7]

2) The values of measured dimensions will be found out using edge detection,following which the values of real dimensions will be found out using (2).

3) A common reference point \& distinct object points will be taken, \& the dimensions will be plotted on the different axes. [3]

4) All these different planes will be then joined at their common edges. For example, considering XY and YZ planes in their respective views, edges with same $Y$ value will be stored as unified XYZ matrix thus converting 2D co-ordinate matrix to 3D co-ordinate matrix. All such planes are joined at their common edges to reconstruct the object as shown in Fig. 4 \& Fig. 5. [1]

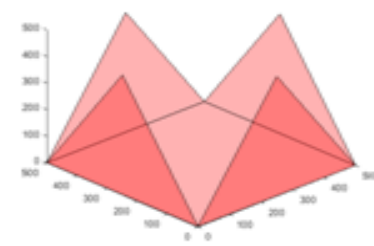

Fig. 4. Before joining common edges

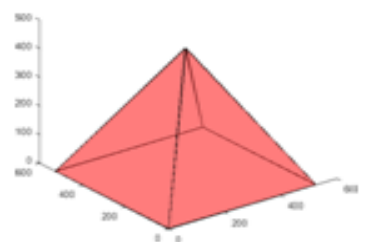

Fig. 5. After joining common edges
Published By:

Blue Eyes Intelligence Engineering and Sciences Publication 


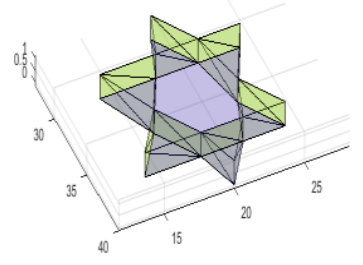

Fig. 6. Reconstruction of a star

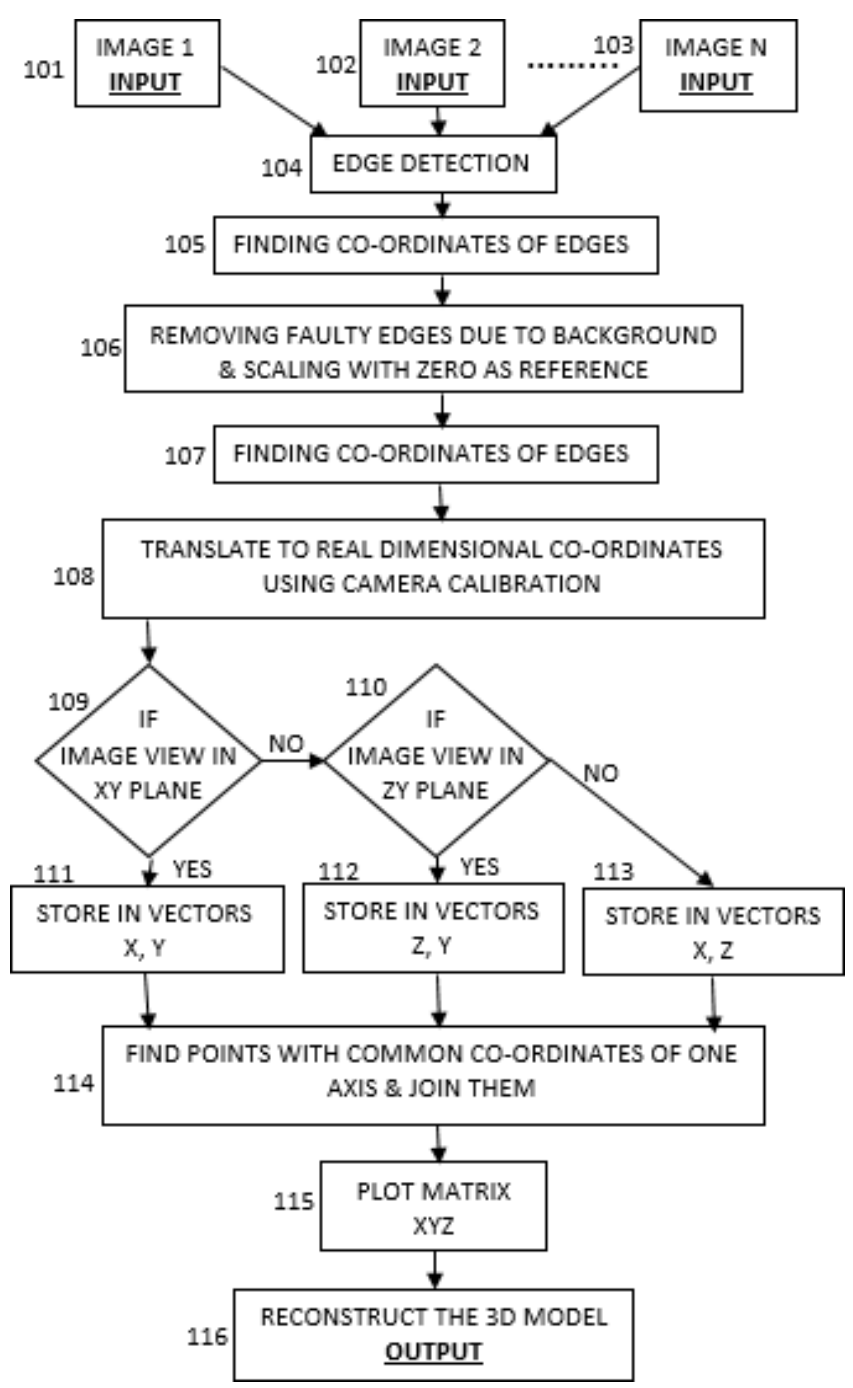

Fig. 8. Flowchart for 3D Reconstruction

\section{Dimensional Analysis, Surface Area \& Volume Estimation}

Dimensional Analysis:

1) Now that the real dimensions from the captured images can be found out, all the real dimensions of the object will be found it.

2) Emphasis will be made on the maximum dimension of the object.

3) Maximum dimension is found by finding the maximum value in the co-ordinate vectorafter scaling it to zero. For example, calculating maximum dimension along $\mathrm{x}$

Surface Area:

1) Now that the model is reconstructed and dimensions are known, triangulation can be performed. [6] [9]

2) Surface of the model can be constructed using such triangles creating a meshmodel.

3) With the known number of triangles on the surface and the dimension of each triangle, the surface area of the object can be calculated as:

Surface Area of the Object $=\Sigma$ (Areas of triangles on mesh)

Volume Estimation:

1) A bounding box will be constructed around the $3 D$ reconstructed object. This bounding box will be in the form of a cube, the side of which will be equal to the largest dimension of the object.

2) The object is now completely inside the bounding box. The area inside the bounding box not occupied by the object (i.e. the space inside the bounding box but outside the object) is called the free space.

3) Small cubes(relative in size to the object) will be built, one by one, adjacent to each other from one corner of the bounding box. These boxes will not intersect the object or each other and will only fill up the free space.

4) This process will go one till all the free space inside the bounding box has been filled.

5) The total volume of the smaller cubes will found out and it will be deducted from the volume of the bounding box. This will give us the volume of the object. Similar method is followed for complex objects. [8]

As seen in Fig. 9, the smaller cubes start filling the bounding box one by one till it is completely filled.

Fig. 10 is the result obtained after the smaller cubes have completely filled the bounding box

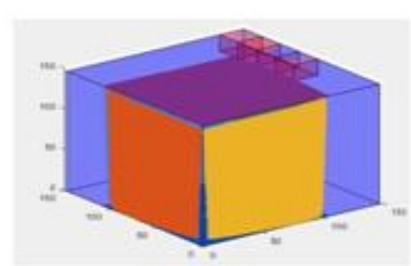

Fig. 9. Beginning of volume estimation

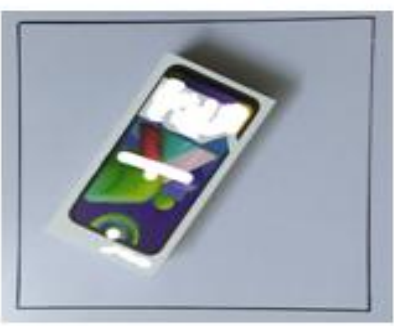

Fig. 11. Top view of object in system

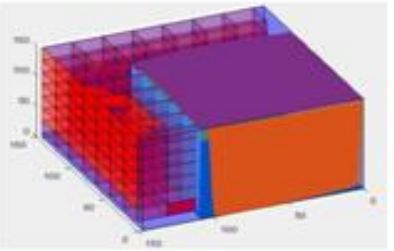

Fig. 10. End of volume estimation

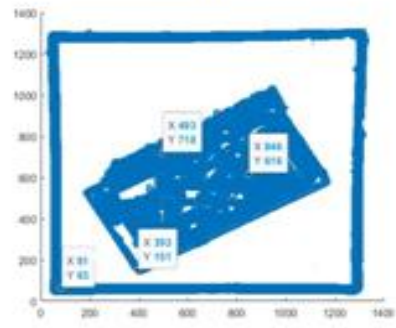

Fig. 12. Co-ordinates of object in system space

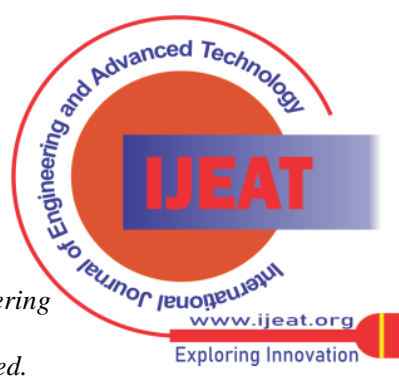




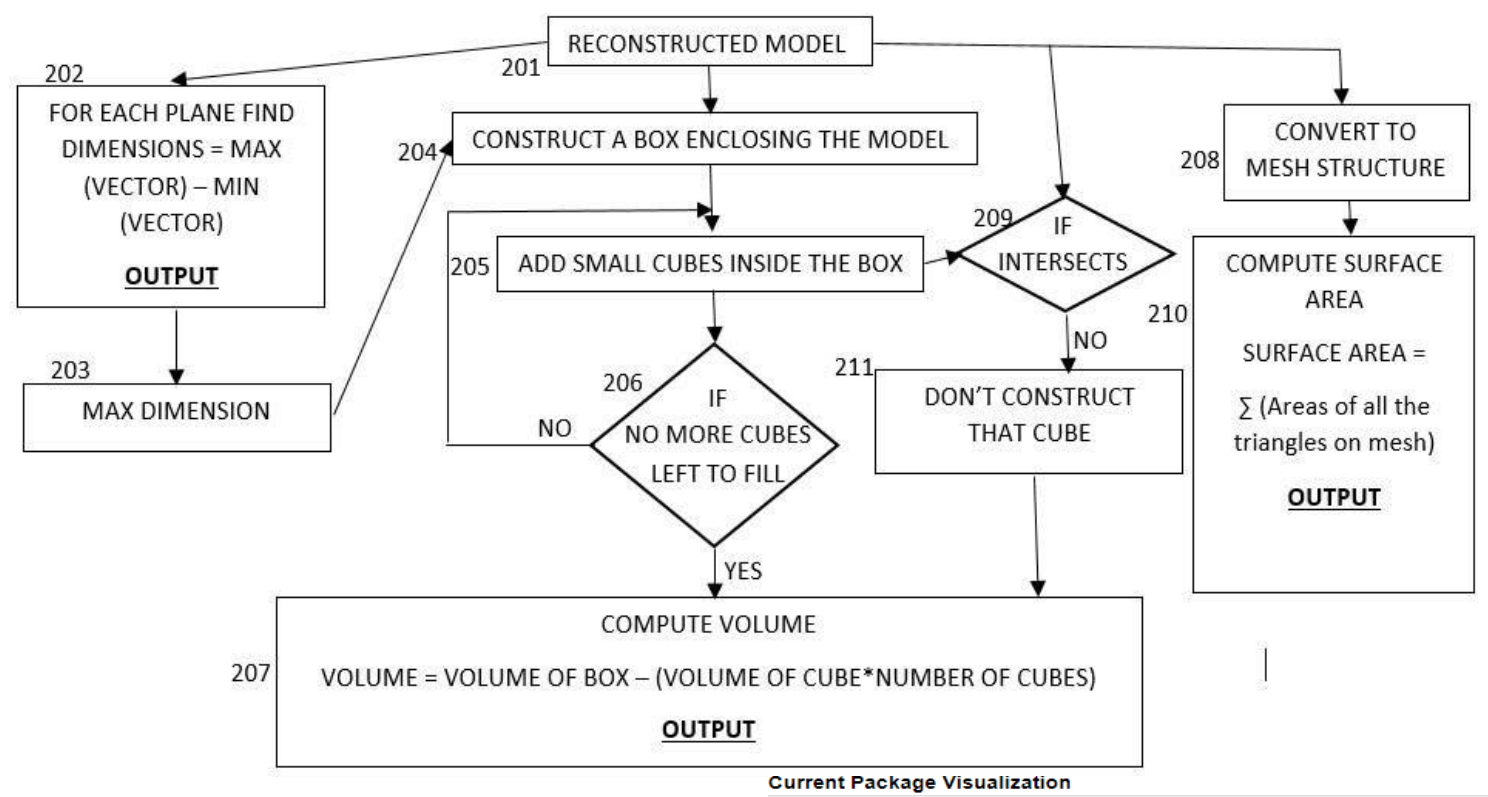

\section{E. Support for Manipulation}

1) After the reconstruction is performed and the $3 \mathrm{D}$ model obtained, the model will be written to a file in the universally accepted stl format.

2) As an added feature, the .stl file will be uploaded to the cloud, on a website set up by us, in an effort to provide decentralized access to the file.

3) This model can even be imported back into our application, once it has been edited and required changes are made. The user will then be able to get the volume estimation of the new edited model.

4) Using the top view image of the object, its exact co-ordinates of where it is placed in the system are found out. For example, in Fig. 14 the black square boundary is the system space where the object, here the mobile casing is placed. Using edge detection of top view as shown in Fig. 15, its co-ordinates can be found out by subtracting the co-ordinates of edges of object after camera calibration by the co-ordinates of the reference point of system space, here it is the bottom most left corner of the square.

5) The changes made by the user in the 3D model in the editor and the co- ordinates of object in system are then used to dictate the changes to the automated machine used to manipulate and make changes in the real object.

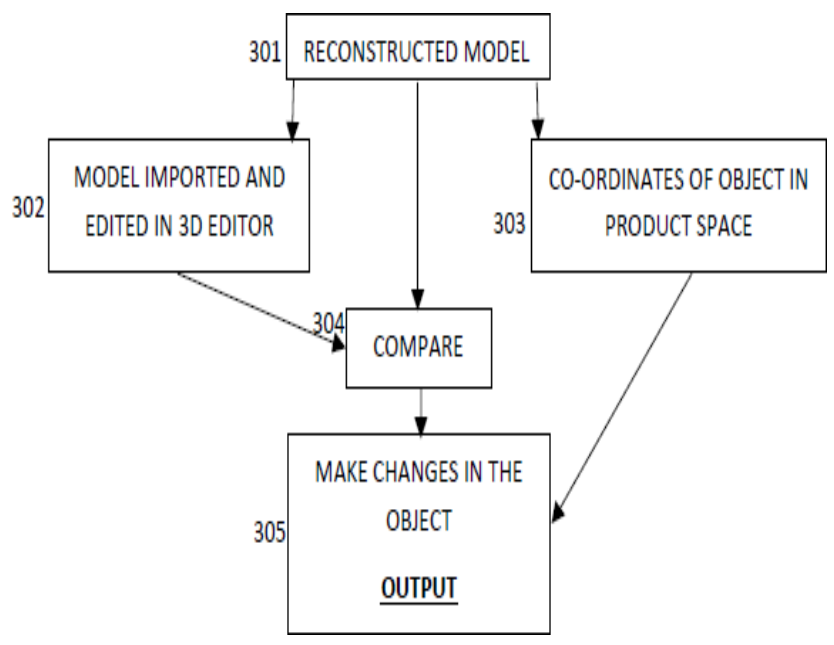

Fig. 14. Flowchart for Manipulation

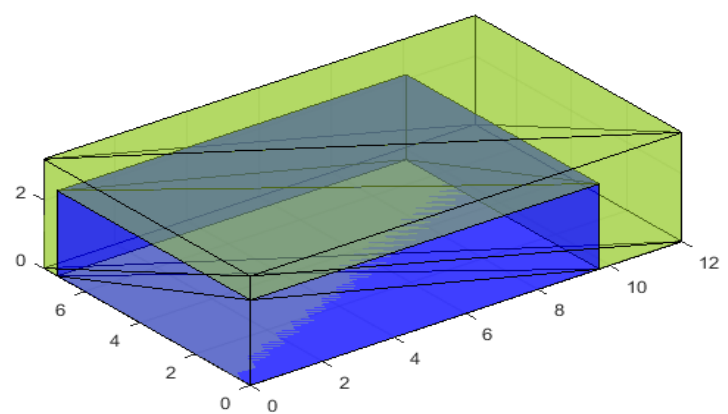

Fig. 15. Object comparison before and after manipulation

6) A comparative report will be compiled, stating the changes made in thenewer edited model from the former model. [10]

\section{RESULT AND DISCUSSION}

For Calibration of camera the observations were made as seen in Table-I.

Table-I: Accuracy in edge detection

\begin{tabular}{|r|r|r|}
\hline $\begin{array}{l}\text { Actual Dimension } \\
(\mathrm{cm})\end{array}$ & $\begin{array}{l}\text { Measured } \\
\text { Dimension }(\mathrm{cm})\end{array}$ & \multicolumn{1}{l|}{ Accuracy (\%) } \\
\hline 6.3 & 6.4042 & 98.346 \\
\hline 6.3 & 6.3455 & 99.2777 \\
\hline 8.5 & 8.6516 & 98.2165 \\
\hline 7.5 & 7.25 & 96.6667 \\
\hline 3 & 3.008 & 99.9733 \\
\hline 3 & 3.0787 & 99.1255 \\
\hline 7.5 & 7.2486 & 96.648 \\
\hline 15 & 15.1208 & 99.1947 \\
\hline \multicolumn{3}{|c|}{ Average Accuracy $=98.4310$} \\
\hline
\end{tabular}

At the end of the dimensional analysis process, output is observed in the format as seen in Fig. 16

Published By:

Blue Eyes Intelligence Engineering and Sciences Publication

(C) Copyright: All rights reserved.

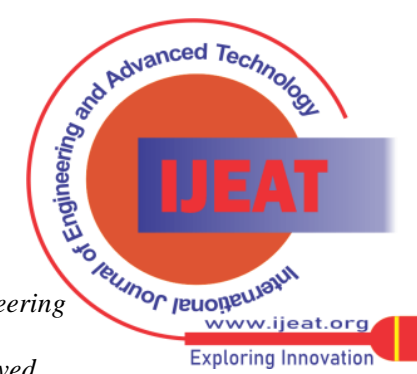




Dimensional Analysis of the Object (in $\mathbf{c m ~} \& \mathrm{~cm}^{\wedge} \mathbf{3}$ )
Max Dimension in X Plane (Length)
25
Max Dimension in Y Plane (Width)
40
Max Dimension in Z Plane (Height)
1
Surface Area of the Object
132.0541
Volume of the Object
33.5000

Fig. 16. Sample Dimensional Analysis

The real dimensions when measured manually by physical methods are tabulated in Table-II.

Table-II: Real Dimensions

\begin{tabular}{|c|c|c|c|c|c|}
\hline \multirow{3}{*}{$\begin{array}{l}\text { Object } \\
\text { Shape }\end{array}$} & \multicolumn{5}{|c|}{ Measured (in cm, $\mathrm{cm}^{2} \& \mathrm{~cm}^{3}$ ) } \\
\hline & \multicolumn{3}{|c|}{ Dimensions } & \multirow[b]{2}{*}{$\begin{array}{l}\text { Surface } \\
\text { Area }\end{array}$} & \multirow{2}{*}{ Volume } \\
\hline & $\begin{array}{l}\text { X } \\
\text { plane }\end{array}$ & Y plane & $\begin{array}{l}\mathrm{Z} \\
\text { plane }\end{array}$ & & \\
\hline Cube & 7 & 7 & 7 & 294 & 343 \\
\hline Cuboid & 15.5 & 13.1 & 12.2 & 1103.94 & 2477.21 \\
\hline Pyramid & 10 & 10 & 6 & 256.2 & 200 \\
\hline Hexagram & 4 & 4 & 2 & 50.476 & 18.476 \\
\hline
\end{tabular}

The computed dimensions when measured using the proposed methodology on captured images are tabulated as seen in Table-III.

\section{Table-III: Computed Dimensions}

\begin{tabular}{|c|c|c|c|c|c|}
\hline \multirow{3}{*}{ Object Shape } & \multicolumn{5}{|c|}{$\begin{array}{l}\text { Computed using proposed methodology } \\
\text { (in } \mathrm{cm}, \mathrm{cm}^{2} \& \mathrm{~cm}^{3} \text { ) }\end{array}$} \\
\hline & \multicolumn{3}{|c|}{ Dimensions } & \multirow[b]{2}{*}{$\begin{array}{l}\text { Surface } \\
\text { Area }\end{array}$} & \multirow{2}{*}{ Volume } \\
\hline & $\begin{array}{l}\mathrm{X} \\
\text { plane }\end{array}$ & Y pl & $\begin{array}{l}Z \\
\text { plane }\end{array}$ & & \\
\hline Cube & 7.13 & 7.05 & 7.02 & 296 & 343.2 \\
\hline Cuboid & 15.8 & 12.9 & 12.6 & 1107.88 & 2477.4 \\
\hline Pyramid & 10.01 & 10.3 & 5.8 & 260.8 & 203 \\
\hline Hexagram & 4.4 & 3.8 & 2.02 & 52.13 & 19.22 \\
\hline
\end{tabular}

After complete testing of our project, the results were observed as seen in Table-IV.

Table-IV: Overall Accuracy

\begin{tabular}{|l|r|}
\hline Process & Accuracy \\
\hline Camera Calibration & $98.43 \%$ \\
\hline 3D Reconstruction (average) & $90 \%$ \\
\hline Dimensional Analysis & $98.91 \%$ \\
\hline Surface Area Estimation & $99.40 \%$ \\
\hline Volume Estimation & $99.10 \%$ \\
\hline
\end{tabular}

As observed in Table-IV, each process has achieved accuracy of $90 \%$ and above.

\section{CONCLUSION}

The paper focused primarily on the need, execution, observations, and analysis of this novel method of 3D Reconstruction, volume estimation and object manipulation. This method is based on and integrated with image processing. The most important advantage of this method is that it doesn't depend on machine learning, which fails to achieve a plausible accuracy due to the lack of a viable dataset. While in some cases machine learning and deep learning can prove more useful, it requires a good amount of hardware processing to perform necessary computations and storage for storing the dataset, if at all available, which is a big disadvantage. This paper discusses of a method which consolidates all the former mentioned processes and automates them completely. The computations that the method performs are not highly resource intensive and are portable i.e. can work on any hardware device. The time taken to perform these operations is lesser than but comparable to similar papers. With the inclusion of IOT for supporting decentralized manipulation will further assist the users of this method to save precious man-hours, especially in the field of manufacturing and research \& development.

It can be observed from the images in this paper, that the features mentioned are indeed achieved with the said level of perfection. The accuracy that this process offers is very high and ever increasing as the methods get further improvement and refinement. Reconstructed models are exact for $90 \%$ of the objects, but for the remaining $10 \%$ object types including complicated curvature shapes it won't be exactly similar to it's object counterpart, until this method is further researched and refined. The accuracy this paper claims is higher than any other paper trying to achieve the similar outcomes, and at the same time also does not use machine learning like some other papers do. Therefore, this paper has achieved its objectives while performing better than any analogous paper.

\section{REFERENCES}

1. Josh Chopin, Hamid Laga \& Stanley J. Miklavcic, "A new method for accurate, high-throughput volume estimation from three 2D projective images", International Journal of Food Properties, vol. 20, issue. 10, pp.2344-2357, 2016, doi: 10.1080/10942912.2016.1236814

2. Liong, Sze \& Gan, Yee-Siang \& Huang, Yen-Chang, "Automatic surface area and volume prediction on ellipsoidal ham using deep learning", Journal of Food Process Engineering, vol. 42, issue. 5, pp. e13093, 2019, doi: 10.1111/jfpe.13093.

3. Ma, Zhiliang \& Liu, Shilong, "Image-based 3D reconstruction techniques and their application in civil engineering", Advanced Engineering Informatics, vol. 37, pp. 163-178, 2018, doi:10.1016/j.aei.2018.05.005

4. C. Yang, F. Zhou and X. Bai, "3D Reconstruction through Measure Based Image Selection", Ninth International Conference on Computational Intelligence and Security, Leshan, pp. 377-381, 2013, doi: 10.1109/CIS.2013.86.

5. S. UluiS,ik, F. Yildiz and A. T. "Ozdem Ir, "Image processing based machine vision system for tomato volume estimation", Electric Electronics, Computer Science, Biomedical Engineerings' Meeting (EBBT), Istanbul, pp. 1-4, 2018, doi: 10.1109/EBBT.2018.8391460

6. W. Qun, X. Zhaohe and W. Jue, "Research and Implementation of 3D Reconstruction Technique Based on Images", 2nd International Conference on Information Science and Control Engineering, Shanghai, 2015, pp. 408-411, doi: 10.1109/ICISCE.2015.96.

Published By:

Blue Eyes Intelligence Engineering and Sciences Publication

(C) Copyright: All rights reserved.

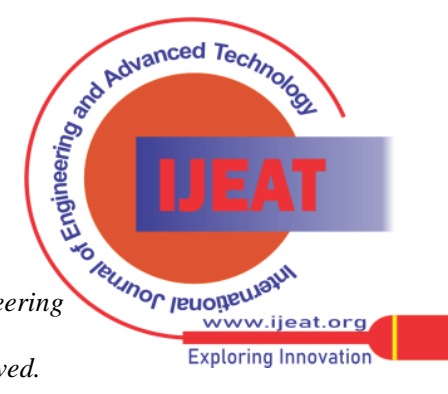


7. M. Li, D. Zheng, R. Zhang, J. Yin and X. Tian, "Overview of 3D Reconstruction Methods Based on Multi-view", 7th International Conference on Intelligent Human-Machine Systems and Cybernetics, Hangzhou, pp. 145-148, 2015, doi: 10.1109/IHMSC.2015.117.

8. B. Presles, J. Debayle, A. Cameirao, G. F'evotte and J. Pinoli, "Volume estimation of 3D particles with known convex shapes from its projected areas", $2^{\text {nd }}$ International Conference on Image Processing Theory, Tools and Applications, Paris, pp. 399-404, 2010, doi: 10.1109/IPTA.2010.5586763.

9. R. Khilar, S. Chitrakala and S. SelvamParvathy, "3D image reconstruction:Techniques, applications and challenges", International Conference on Optical Imaging Sensor and Security (ICOSS), Coimbatore, pp. 1-6, 2013, doi:10.1109/ICOISS.2013.6678395.

10. Kholgade, Natasha \& Simon, Tomas \& Efros, Alexei \& Sheikh, Yaser, "3D Object Manipulation in a Single Photograph using Stock 3D Models", ACM Transactions on Graphics. vol. 33, issue. 4, pp. 1-12, 2014, doi: 10.1145/2601097.2601209.

\section{AUTHORS PROFILE}

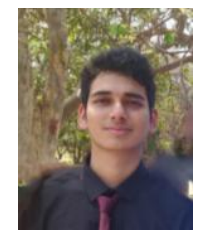

Soham Pinge, was born in Mumbai, India in 2000. He is currently a final year student in the Department of Electronics and Telecommunication Engineering, Sardar Patel Institute of Technology (S.P.I.T), Mumbai. His research interests include IOT, cloud computing, data \& business analytics, industrial solutions, machine learning and embedded systems.

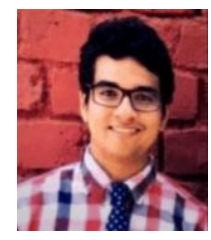

Nitish Wadhavkar, was born in Pune, India in 2000. He is currently a final year student in the Department of Electronics and Telecommunication Engineering, Sardar Patel Institute of Technology (S.P.I.T), Mumbai. His research interests include embedded systems, mathematics and computing, industrial solutions, IOT, Machine learning and data analytics.

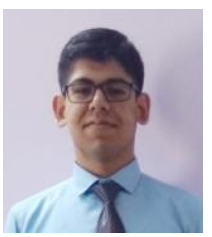

Amey Singh, was born in Mumbai, India in 1999. He is currently a final year student in the Department of Electronics and Telecommunication Engineering, Sardar Patel Institute of Technology (S.P.I.T), Mumbai. His research interests include cloud computing, IOT, Machine learning.

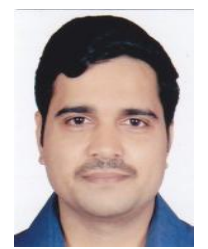

Dr. Amol Deshpande, was born in Aurangabad, India, in 1984. He received B.E and M.Tech degrees in Dr. Babasaheb Ambedkar Marathwada University, India. He received the Ph.D. degree in Electrical engineering from Veermata Jijabai Technological Institute (VJTI), Mumbai, India in 2014. He has worked as a Post Doctoral Fellow in Institute for Plasma Research (IPR), Gandhinagar, India. He joined the Department of Electronics and Telecommunication Engineering, Sardar Patel Institute of Technology (S.P.I.T) as an Assistant Professor. His current research interests include Image Processing, Non-Destructive testing, Partial Discharge, High Voltage Engineering, Pulsed Electric Fields. He has published several research papers in reputed Conferences \& Journals.

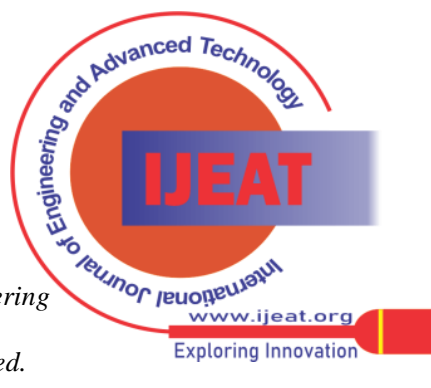

